\title{
L'administration du Travail et les conflits collectifs : Citroën et Talbot (1982-1983)
}

Labour Administration and collective Conflicts : Citroën and Talbot (1982-1983)

\section{Claude Chetcuti et Nicolas Hatzfeld}

\section{(2) OpenEdition}

1 Journals

Édition électronique

URL : http://journals.openedition.org/travailemploi/4631

DOI : 10.4000/travailemploi.4631

ISSN : 1775-416X

Éditeur

DARES - Ministère du Travail

Édition imprimée

Date de publication : 30 juin 2007

Pagination : 31-38

ISSN : 0224-4365

\section{Référence électronique}

Claude Chetcuti et Nicolas Hatzfeld, "L'administration du Travail et les conflits collectifs : Citroën et Talbot (1982-1983) », Travail et Emploi [En ligne], 111 | juillet-septembre 2007, mis en ligne le 15 septembre 2009, consulté le 01 mai 2019. URL : http://journals.openedition.org/travailemploi/4631 ; DOI : 10.4000/travailemploi.4631 


\title{
L'administration du Travail et les conflits collectifs : Citroën et Talbot (1982-1983)
}

\author{
Claude Chetcuti (*), Nicolas Hatzfeld (**)
}

\begin{abstract}
Les violents conflits dans les usines Citroën et Talbot qui accompagnèrent l'arrivée de la gauche au gouvernement en 1982-1983 sont présentés ici à la fois sous l'angle historique et sous l'angle du témoignage. Le premier volet de l'article est rédigé par un historien: il fait le récit circonstancié de ces luttes et illustre le mouvement des ouvriers spécialisés qui connaîtront à cette occasion un profond changement d'image. Le second laisse la place au témoignage vécu du directeur régional d'Île-de-France d'alors: tirés de deux entretiens entre les deux auteurs menés les 20 septembre et 28 novembre 2006, ces extraits mettent l'accent sur les modes d'intervention de l'administration du travail dans ces conflits. Un troisième conclut sur leur inscription dans le mouvement social et sur leur portée historique.
\end{abstract}

En mai et juin 1982 éclatent à Citroën et à Talbot-Poissy des conflits du travail d'une gravité et d'un retentissement peu communs. S'ils s'inscrivent dans un mouvement d'ouvriers spécialisés qui affecte alors toutes les entreprises automobiles, ils comportent, chez Talbot comme chez Citroën, des revendications qui ont fait parler de «printemps de la dignité » ou de «printemps syndical» (BENoit, 1982). Un an plus tard, les enjeux se déplacent et marquent un changement d'époque. Les suppressions d'emploi touchent l'entreprise-mère PSA puis l'ensemble de la branche automobile, se traduisant, notamment, par un second conflit à Poissy, aussi violent que le premier, dans l'hiver 1983-1984(1). Ces deux temps d'affrontement, qui accompagnent l'arrivée de la gauche au gouvernement puis son tournant de 1983 vers une politique de rigueur, affectent les forces politiques, les partenaires sociaux et les pouvoirs publics. Ils mettent à l'épreuve les modes d'intervention de l'administration du travail dans les conflits sociaux. Les grèves de 1982 amènent en effet le ministère à mettre en œuvre, d'abord à Citroën puis chez Talbot de façon presque identique, des procédures exceptionnelles pour leur résolution et à effectuer un suivi des décisions qui détonne d'avec la tradition d'action ponctuelle. Dans le second temps, le conflit Talbot de l'automne-hiver 1983-1984 amorce une période où la nécessité d'intervenir face aux suppressions d'emploi accentue l'intervention des pouvoirs publics dans les conflits.

La contribution qui suit examine ces questions de trois façons différentes. Tout d'abord, suivant une chronologie courte, les conflits sont examinés dans leur contexte économique, social et politique, qui

(*) Inspecteur général honoraire des affaires sociales, alors directeur régional du travail d'Île-de-France.

(**) Maître de conférences en histoire contemporaine, université d'Evry. Nicolas.hatzfeld@wanadoo.fr

(1) Peugeot société anonyme. leur donne toute leur portée. Après ce temps d'histoire, la focale se resserre sur l'action de l'administration du travail tandis que l'écriture historienne laisse la place au témoignage de Claude Chetcuti, qui fut directement impliqué dans cette action en tant que directeur régional du travail d'île-de-France. Enfin, de nouveau dans le registre historien, le texte reprend l'action de l'administration du travail dans les conflits, cette fois dans une perspective de longue durée, à la fois pour faire ressortir le cheminement français des lois et pratiques en la matière et pour proposer une lecture du moment que constitue le règlement des conflits Citroën et Talbot.

\section{Les conflits Citroën et Talbot}

De nombreuses usines de l'industrie automobile sont touchées par des conflits entre 1981 et 1983. Parmi elles, les deux acquisitions récentes du groupe PSA que sont Citroën et Talbot, entrelacent des discordances sociales, d'entreprise, économiques et politiques dont les évolutions conduisent à des grippages de forte intensité.

\section{Citroën et Talbot, 1982: mouvement d'ouvriers spécialisés et revendications de liberté}

Lorsque surviennent les grèves de 1982, dans les deux entreprises règne un système social d'exception constitué au milieu des années 1950 à Simca et quelques années plus tard chez Citroën. Plus que par la présence d'un syndicat corporatiste, Confédération française du travail puis Confédération des syndicats libres (CSL), accueillant tous les types de salariés, de l'ouvrier spécialisé à l'ingénieur, ce système se caractérisait par une sorte d'intégration des fonctions de gestion du personnel et de maintien de l'ordre intérieur, de représentation syndicale et de prise en charge des œuvres sociales. Le système prétendait à l'exclusivité et combattait avec énergie les syndicats 
représentatifs : jusqu'au milieu des années 1970, la CGT de l'usine de Poissy vivait dans la clandestinité(2). Dirigé par des hommes liés aux partis de droite et d'extrême droite, auxquels il fournissait des colleurs d'affiche et parfois des candidats aux élections locales, il prolongeait une tradition de la droite sociale française (FAVRE, 1998). Après 1968, la formule avait tenté d'autres entreprises, telles que Peugeot, sans s'instaurer en système. Cette formule connaissait cependant des difficultés croissantes au cours des années 1970. Tout d'abord, pour délivrer d'importants avantages sociaux(3) distribués selon une formule clientéliste, il fallait un budget considérable, dont le déséquilibre incessant supposait une croissance continue. PSA, qui avait racheté ces entreprises (4), craignait pour elle-même leurs dispositifs opaques. Ensuite, l'État - l'administration et la justice - était de plus en plus porté à faire entrer ces territoires spécifiques dans le droit commun. À Poissy, les élections professionnelles entachées d'irrégularités entraînaient depuis 1978 des procédures judiciaires et la suspension des suivantes(5). Au début de 1981, une décision du juge des référés de Bobigny chargeait des membres du Conseil d'État de surveiller les élections professionnelles (LOUBET, HatzFeld, op. cit., pp. 230-231). Après les élections de 1981, le statu quo devient intenable chez Talbot et Citroën : la persistance des fiefs CSL contrecarre la politique du ministre du Travail Jean Auroux qui prépare des lois donnant de nouveaux droits aux travailleurs, votés dans la seconde moitié de 1982 (BECKER, 1998). La préparation des élections professionnelles de 1982 s'effectue dans une tension qui inquiète le gouvernement (6).

Le 22 avril, des milliers d'ouvriers d'Aulnay, comme dans les autres usines Citroën de la région parisienne, se mettent en grève et placent des piquets aux portes de l'usine (7). Les revendications concernent les cadences, la réforme des classifications et la

(2) En 1960, la CGT ne comptait que seize adhérents, organisés en triangle comme certains réseaux de résistants ( $c f$. TREHEL, 1982; Loubet, HatzFeld, 2002).

(3) Simca avait ainsi créé une retraite complémentaire confortable, conçue pour être alimentée par le turn over d'ouvriers spécialisés généralement étrangers ( $c f$. Loubet, HatzFeLd, op. cit., pp. 116-124 et 232).

(4) La crise des années 1970 a conduit Peugeot à reprendre Citroën en 1974 et Chrysler-France en 1978 (Loubet, 2001).

(5) «En raison d'un "climat socialement malsain", les tribunaux proposent un contrôle des élections professionnelles chez Talbot (Poissy)», Le Monde, 4 janvier 1980.

(6) La CFDT alerte les ministères sur des agressions subies par certains militants à Citroën Aulnay. Elle est reçue par un conseiller du Premier ministre [CFDT, archives de la FGM et de la Fédération des mineurs (1917-1988), carton 1B 417]. À Talbot Poissy, la direction accepte la présence d'inspecteurs du travail lors des élections, qui seront reportées compte tenu du conflit.

(7) Quelques films militants peuvent éclairer, à leur façon, le conflit de Citroën en 1982. Ainsi, Distribution de tracts devant les usines Citroën, anonyme, 1975; Le mariage de Monsieur CGT avec Madame Citroën ou comment la lutte des classes vint à Ghazi Akka, de Jean-Patrick Lebel, 1982; Haya, de Claude Blanchet et Édouard Bobrowski, 1982. formation professionnelle, comme à Billancourt, Flins ou Sochaux et renvoient à l'évolution de la condition des ouvriers spécialisés. Elles portent aussi sur les salaires et la possibilité pour les immigrés de joindre la cinquième semaine aux congés d'été. Mais, particularité de l'entreprise, les grévistes revendiquent les libertés au travail et la reconnaissance des droits syndicaux. Le directeur régional du travail d'île-deFrance établit des contacts bilatéraux avec la direction des usines et les syndicats tandis que des relations analogues ont lieu au niveau ministériel voire à celui du Premier ministre. Durant un mois, les négociations piétinent tandis que fleurissent les manœuvres de terrain et les bagarres (8). Puis, le 21 mai, est annoncée la nomination du professeur Jean-Jacques Dupeyroux comme médiateur. Après qu'il ait écouté les différentes parties, sa recommandation, rédigée le 26, est acceptée le 27 par les partenaires sociaux. Elle porte sur les «problèmes relatifs aux libertés et, de façon plus générale, au respect de la dignité de chacun "comme sur" les problèmes plus classiques relatifs aux salaires et aux conditions de travail(9)». La recommandation prévoit également la création de commissions de suivi des dossiers qui ne peuvent trouver de solution immédiate: une commission relative aux libertés et une autre traitant des salaires et classifications, présidées respectivement par Pierre Bois, inspecteur général du travail, et Claude Chetcuti, directeur régional. La production reprend le $1^{\text {er }}$ juin.

Aussitôt, le mercredi 2 juin 1982, le travail s'arrête à l'usine Talbot de Poissy dans l'atelier regroupant les lignes d'assemblage, sur la base d'une plate-forme revendicative semblable à celle d'Aulnay-sous-Bois. Le débrayage s'impose malgré une intervention musclée de la CSL. Le lendemain après-midi, un affrontement très violent fait plus d'une quarantaine de blessés, dont le directeur du personnel. Les grévistes restent maîtres du terrain, tandis que dans les autres bâtiments, le travail reste dominant. Ici aussi, tandis que l'administration du travail s'emploie dans l'usine à rétablir des liens, comme le montre plus loin le témoignage de Claude Chetcuti, des contacts sont pris entre la direction du travail et la direction de PSA et les ministères, Matignon, voire l'Élysée; ils se poursuivront tout au long de la grève. Dès lors, et pendant quatre semaines, le conflit s'installe dans la durée, opposant près de 7000 grévistes totaux ou partiels à 10046 non grévistes (10). Le 23 juin, le ministre du Travail désigne à nouveau comme médiateur JeanJacques Dupeyroux, qui remet ses recommanda-

(8) «Guerre civile en Île-de-France», écrit sous un pseudonyme un directeur de Citroën dans le livre: Ewald, L'école des esclaves, Paris, éditions de la Table ronde, 1983.

(9) Conflit Citroën, Recommandation du médiateur, 26 mai 1982. CFDT, archives de la FGM et de la Fédération des mineurs (1917-1988), carton 1B 417.

(10) Plus de 1000 salariés sont considérés comme grévistes à $100 \%$, et 5415 subissent des retenues de salaires de 10 à $90 \%$. Archives PSA Peugeot-Citroën. 
tions le $1^{\text {er }}$ juillet: elles reprennent celles présentées chez Citroën, portant sur les droits et les libertés, la dignité des travailleurs, le droit syndical, la création de lieux de culte, les conditions de travail et enfin les modalités de reprise du travail. Pour les rémunérations, le blocage des prix et des salaires imposés par le gouvernement reporte au $1^{\text {er }}$ novembre 1982 l'attribution d'une prime générale et d'une augmentation des taux. La médiation prévoit également la création de commissions sur les libertés et sur les salaires, les carrières et la formation des ouvriers spécialisés, présidées par Roger Salmon, inspecteur général, et André Nutte, directeur départemental des Yvelines. La CGT prend position pour la reprise du travail, qui est votée le 2 juillet à une écrasante majorité(11). Le 3 juillet, la direction de Talbot accepte les recommandations du médiateur.

Dans les deux conflits, le système corporatiste se brise, même si une partie des salariés n'a pas fait grève et si la CSL reste très influente. Les élections de juin aux usines Citroën de la région parisienne voient la CGT bondir de 9 à $57 \%$, la CFDT atteindre $6 \%$, tandis que la CSL tombe de $82 \%$ à $33 \%$. À Poissy, la CGT réalise une syndicalisation massive et annonce 3327 adhérents le 2 juillet. La CFDT suit aussi une forte progression, quoiqu'à bonne distance. Chez Citroën comme chez Talbot, la reprise est marquée par des tensions, à propos de variations de cadence, de mutations, de salaires, ou de relations entre ouvriers et agents de maîtrise. À Poissy, à côté des formes classiques de représentation, la CGT et la CFDT instaurent un réseau de délégués de chaîne, issus des actifs de l'occupation (HATZFeld, 2003). Au-delà de ces vagues immédiates et des reflux ultérieurs, Citroën et Talbot amorcent une harmonisation progressive mais irréversible des relations sociales avec la France du travail des années 1980.

\section{3: retour de la crise industrielle, nouveau conflit et changements d'image}

Cependant, la branche automobile connaît un retour en force des difficultés amorcées par la seconde crise de 1979. Dès cette date, les ventes des deux grands constructeurs se sont affaissées (LOUBET, op. cit., pp. 401-418), PSA perdant de l'argent dès 1980 et Renault l'année suivante. La gravité de cette crise a été sous-évaluée par les entreprises, les syndicats et le pouvoir politique. Jusqu'en 1981, les syndicats ont hésité à parler de crise (12) tandis que les directions d'entreprise ont imaginé, comme en 19731974, un redémarrage des marchés. La victoire de la gauche a perturbé les diagnostics (LOUBET, op. cit. p. 414), mais les pertes se sont accumulées et les

(11) Les chiffres de la direction notent 3973 votants, 3698 oui, 262 non et 13 nuls. Archives PSA Peugeot-Citroën, site de Poissy.

(12) Archives de la FGM et de la Fédération des mineurs (1917-1988), carton 1B 417. commandes s'effondrent fin 1982. Avec l'arrivée de Jacques Calvet, PSA(13) s'engage dans une réduction drastique des charges et des effectifs. Plus lourdement endetté, Renault perpétue son rôle de symbole industriel et social(14), et ne réagira énergiquement qu'en 1985 avec l'arrivée de Georges Besse. Entre-temps, la gauche opère son revirement stratégique dans les derniers jours de mars 1983. Le 21, le franc est dévalué pour la troisième fois. Le 23, François Mitterrand annonce la nouvelle politique économique du gouvernement Mauroy; le 25, Jacques Delors présente son plan, avec en point d'orgue le grand virage de la rigueur.

Chez PSA, Poissy est la branche la plus en difficulté. Talbot a réduit ses effectifs dès 1979, en utilisant tous les moyens: départs à l'amiable, licenciements économiques, aide au retour pour les travailleurs immigrés volontaires. Deux plans successifs établis avec le Fonds national pour l'emploi (FNE) ont éloigné des salariés âgés de 56, puis de 55 ans. Au début 1983, les commandes de Talbot s'effondrent, les jours chômés se multiplient et les salariés s'inquiètent sur l'avenir du site et de l'emploi. De fait, le 12 juillet 1983, la direction de PSA annonce 4140 nouveaux départs soit un quart des emplois de Poissy, dont 1235 mises en préretraite par convention FNE et 2905 licenciements. Dans l'usine, l'émotion est très vive. Les pouvoirs publics tentent d'éviter de devoir avaliser des suppressions d'emploi. Malgré les relations très froides entre l'entreprise et le gouvernement, des contacts s'établissent entre Jacques Calvet et Pierre Bérégovoy, ministre des Affaires sociales. Mais le 12 septembre, la direction de Talbot dépose une demande de licenciements. Le gouvernement en refuse les modalités sociales, demande une réduction du nombre des licenciements, et des engagements sur l'avenir du site de Poissy. Le 21 novembre, la direction de Talbot renouvelle son plan de licenciements en aménageant le volet social et offre des garanties sur l'avenir de l'usine. Entre-temps, les contacts se multiplient entre syndicats, instances de l'entreprise, ministères, autorités départementales et élus. Le dossier Talbot constitue une épreuve de vérité pour le nouveau réalisme économique du gouvernement, qui repousse la décision. Les partenaires perdent patience, une grève avec occupation se déclenche le 7 décembre 1983 tandis que la direction de PSA met en balance la survie de Poissy et annonce la suspension des rémunérations. Le 17 décembre, le Premier ministre Pierre Mauroy autorise 1905 licenciements.

(13) Arrivé chez PSA au printemps 1982, Jacques Calvet est nommé à la présidence du conseil de surveillance d'Automobiles Peugeot le 17 novembre.

(14) Pierre Dreyfus, ancien PDG de la Régie, est ministre de l'Industrie de juin 1981 à juin 1982. 
Les conditions de départ sont améliorées(15), tandis qu'un plan de départs en préretraite est signé. Pour moderniser Poissy, PSA promet 1,2 milliard de francs d'investissements. Toutefois, le personnel est tiraillé entre des jusqu'au-boutistes du refus des licenciements et des tenants de la relance de l'usine à tout prix. Du 3 au 5 janvier, les ateliers sont le théâtre d'affrontements d'une violence exaspérée entre les deux camps (Loubet, HatzFeld, op. cit., pp. 262-264) qui font plus de 100 blessés dont 18 hospitalisés. Seule l'intervention des CRS, souhaitée par tous, rétablit le calme. Quelques jours plus tard, l'usine reprend le travail, définitivement. Presque parmiracle, les anciens protagonistes recommencent à travailler ensemble et Poissy amorce son redressement industriel.

D'un bout à l'autre des conflits de l'automobile des années 1982-1983, la représentation des ouvriers immigrés dans l'espace public se modifie considérablement. Tout d'abord, les images de grève font voir à tous l'importance de ces ouvriers immigrés dans les usines automobiles Citroën et Talbot, après celles de Flins et de Billancourt (PITTI, 2002; BECKER, op. cit., p. 296). De fait, la tendance à recruter des ouvriers d'origine étrangère, forte dans les usines de l'agglomération parisienne, a été reprise par la plupart des usines de province dans les années 1970. Pourtant en 1982, lors des grèves de Renault comme des conflits de Citroën, c'est essentiellement comme ouvriers que ces grévistes sont présentés dans une grande partie de la presse. Ouvriers spécialisés d'abord, et parfois immigrés. L'émergence du Marocain Akka Ghazi en animateur de la grève d'Aulnay illustre l'ancrage du syndicalisme dans cette nouvelle génération ouvrière, et l'intégration de celle-ci dans la société française. André Sainjon, responsable de la fédération des métaux CGT refuse de distinguer ces ouvriers spécialisés comme immigrés(16). Les recommandations du médiateur ne désignent que secondairement les ouvriers spécialisés comme immigrés et sont centrées sur «l'immense question de la condition des $\mathrm{OS} \gg$. Elles évoquent aussi, à plusieurs reprises l'éventualité de discriminations xénophobes, la nécessité de combattre le racisme et la mise à disposition de lieux de culte dans l'enceinte de l'usine. Toutefois la spécificité immigrée de ces ouvriers spécialisés se dessine dans une perspective d'intégration et en second plan. Dans cette perspec-

(15) Elles comportent l'attribution d'une prime de 20000 francs à tout employeur procédant à une réembauche, une aide à la création d'entreprise, ainsi qu'une réduction de 20000 francs sur l'achat d'un véhicule utilitaire servant d'outil de travail. Des dispositifs de formation ou de reclassement sont mis en place: 1300 stages de formation rémunérés et 500 reclassements dans d'autres entreprises, PSA prenant en charge la formation d'une centaine de salariés aux métiers de la réparation automobile. ( $c f$. Loubet, HATZFeld, op. cit., pp. 259-260).

(16) André Sainjon, secrétaire général de la Fédération des travailleurs de la métallurgie CGT, préface à Floriane Benoit, op. cit. tive, la commission présidée par Gabriel Ducray (17) cherche à décloisonner la condition d'OS, et souligne la nécessité d'entreprendre l'alphabétisation de la majorité des intéressés pour permettre de leur proposer une carrière professionnelle(18). Et pour cause: à Poissy, l'illettrisme était décisif pour les recrutements effectués prioritairement dans les campagnes marocaines, de sorte que de jeunes citadins scolarisés allaient y candidater après s'être râpé les mains dans le sable afin de les rendre plus rustiques (LOUBET, HATZFELD, op. cit., pp. 192-198).

En janvier 1983, à l'occasion de grèves chez Renault, la perspective change. Pierre Mauroy stigmatise «des travailleurs immigrés [...] agités par des groupes religieux et politiques qui se déterminent en fonction de critères ayant peu à voir avec les réalités sociales françaises » (19). Au même moment, Gaston Defferre, ministre de l'Intérieur, parle «d'intégristes, de chiites». Les phrases sont remarquées(20) et traduisent un glissement: la Gauche avait abrogé en octobre 1981 la loi dite Bonnet-Stoleru, supprimé l'aide au retour et aidé au regroupement familial afin de permettre l'intégration dans la société française des immigrés qui le désiraient (BECKER, op. cit., pp. 280-281). À côté des thèmes de sureffectifs et de main-d'œuvre formée et adaptable, l'accent mis sur le caractère étranger et peut-être inintégrable des ouvriers immigrés recompose les argumentaires de la crise. En décembre, Jack Ralite, ministre délégué chargé de l'emploi, annonce à l'Assemblée nationale que les immigrés de Talbot seront «aidés, s'ils en expriment le souhait, à retourner dans leur pays (21)». L'immigré a supplanté l'ouvrier spécialisé, l'autre pays n'est plus seulement celui des origines. Le débat est vif entre syndicalistes, et entre salariés. Pour les ouvriers immigrés eux-mêmes la question du chez soi est longtemps restée ouverte: derrière le retour souvent projeté, l'avenir français des enfants, jamais évoqué par les politiques, a modifié insensiblement les repères. La sommation de choisir attise la douleur du choix.

(17) Gabriel Ducray avait été inspecteur du travail, directeur du CEREQ de sa fondation en 1969 à 1980 puis délégué à la formation professionnelle, avant de devenir membre de la délégation française à l'OIT.

(18) Commission d'étude pour le développement de la formation des ouvriers spécialisés, Travail et formation des ouvriers de fabrication de l'industrie automobile, octobre 1983. Archives Gerpisa, université d'Évry. L'étude centre le problème sur les ouvriers spécialisés, mais évoque modérément le lien entre la structure de l'emploi et l'organisation du travail de fabrication dans ces grandes usines automobiles qui reposent sur une maind'œuvre jeune vouée à la mobilité externe ou interne. Un système grippé par l'arrêt des embauches, qui immobilise les trajectoires. (19) Pierre Mauroy, interview à Nord-Eclair. Cité par Le Monde, 29 janvier 1983.

(20) Michel Noblecourt, «Une phrase de trop», Le Monde, 30-31 janvier 1983; "Immigrés et islamisme: quelle mouche a piqué Mauroy et Defferre?», Libération, $1^{\text {er }}$ février 1983.

(21) Le Monde, respectivement 30 et 31 janvier 1983, et 23 décembre 1983. 
Pour conclure, quelques brèves remarques. Les conflits Citroën et Talbot de 1982-1983 traduisent, par leur intensité, un tissage spécifique des liens sociaux et économiques, qui ne résiste pas aux tensions qu'entraînent ces temps de crise. À travers eux se joue tant un moment de réforme des relations sociales en entreprise que la prise en compte, par les acteurs sociaux, des recompositions de l'emploi. Au-delà de l'importance médiatique, la portée de ces épreuves tient aussi à l'imbrication des échelles, de l'atelier à la dimension nationale. Les systèmes Simca et Citroën s'effondrent lorsque l'État leur retire son soutien, soucieux de renforcer l'expression des salariés dans leur entreprise. Plus tard, les menaces sur l'usine de Poissy forcent la gauche à étendre à l'emploi son aggiornamento économique. Des ouvriers vieillissants jusqu'au Premier ministre, tous les protagonistes réorientent leurs positions et voient leur rôle se redéfinir.

\section{L'action publique,}

\section{entre conciliation et médiation}

\section{Témoignage de Claude Chetcuti, alors directeur régional du travail en Île-de-France (22)}

«À Citroën, le conflit de 1982 démarre, dans mon souvenir, sur une revendication de salaire. C'est au début un conflit assez classique. Mais il se double très vite d'une revendication syndicale et d'une revendication de dignité de la part de la masse des ouvriers qui sont encadrés par la CGT [...]. Là-dessus, comme dans le conflit de Poissy mais de façon moins violente, il se produit une incapacité pour les deux parties de se rencontrer. Je me souviens fort bien d'avoir été à l'usine de Javel, pour rétablir un minimum de discussion ou de dialogue, qui se faisait par micros interposés. Il n'y avait pas de contacts physiques (23). Ici intervient une difficulté fondamentale, l'impossibilité de rassembler, de mettre face à face des interlocuteurs. Finalement, l'échec des mini-négociations s'est traduit par un recours à une médiation de Jean-Jacques Dupeyroux, qui avait été le conseiller officiel ou officieux de différents ministres du Travail depuis plusieurs années (il avait notamment remis à Jean Mattéoli un rapport sur la lutte contre le travail illégal). C'est une des rares occasions où cette procédure a été appliquée. [...]. Ce qui m'a paru caractéristique, c'est que ce conflit s'est noué à des niveaux très élevés. La première réunion que j'ai menée avec la CGT s'est déroulée jusqu'à minuit, à la Direction régionale du travail, avec le secrétaire national de la Fédération des métaux André Sainjon. Je n'ai jamais fait de rapport écrit, mais j'étais tous les jours, sinon

(22) Entretiens avec Nicolas Hatzfeld, les 20 septembre et 28 novembre 2006.

(23) Dans son livre Citroën, Le printemps de la dignité..., op. cit., p. 28, Floriane Benoit fait état d'une rencontre se déroulant le 15 mai quai de Javel, par l'intermédiaire d'un système vidéo, chaque délégation étant isolée dans une salle. plusieurs fois par jour, en liaison avec un membre du cabinet du ministre. [Du côté syndical donc], ce n'étaient pas les gens du terrain qui négociaient, c'était la Fédération des métaux, mais au niveau confédéral. Finalement, pour Citroën, tout cela s'est dénoué en présence de $\mathrm{M}$. Cornu, membre du cabinet du ministre, et de moi-même, dans l'appartement de M. Dupeyroux, où André Sainjon a accepté de donner son aval à la médiation de début juin.

À peine le conflit chez Citroën s'était-il terminé que survenait le mouvement à Poissy. Le Journal officiel s'en est fait l'écho, puisque le jeudi 3 juin ont lieu ces mouvements de grève violents au cours desquels un cadre est blessé. Le problème a été presque immédiatement évoqué à la chambre, où Jean Auroux a déclaré qu'il chargeait le directeur du travail et le directeur régional du travail d'Île-deFrance, donc monsieur Chazal et moi-même, d'intervenir et de se rendre immédiatement sur place. Il y avait là le même type de revendications qu'à Citroën, même si ce n'était pas tout à fait le même type de population et si les immigrés, en proportion plus forte à Poissy, étaient beaucoup plus encadrés et pris en charge par l'entreprise, qui les logeait dans des foyers beaucoup plus fréquemment que ce n'était le cas chez Citroën. Dans mon souvenir, l'intervention était assez ardue. Après l'affrontement physique violent, il y avait une rupture totale entre les deux parties. La première difficulté qu'on a eue, et qui a duré tout le samedi, a été d'arriver à réunir les deux parties autour d'une table, ce qui s'est fait à la sous-préfecture de Saint-Germain-en-Laye, en terrain neutre. Quand finalement on a obtenu cela, Monsieur Chazal a jugé que ce n'était plus du domaine de l'administration centrale et m'a confié le suivi de l'affaire. Là, tous les jours pendant une dizaine de jours, j'ai réuni les parties matin et soir, ou plutôt tous les après-midis, mais cela durait quelquefois jusqu'à 22 heures ou 24 heures à la souspréfecture de Poissy. Ça a été un dialogue de sourds pendant un certain nombre de journées où j'ai passé mon temps à essayer de mettre les gens en place. J'ai noté les heures passées à la sous-préfecture durant les premiers jours du conflit.

La conciliation sur le terrain : présence à TalbotPoissy aux premiers jours du conflit (24)

$\begin{array}{lll}\text { Vendredi } 4 \text { juin } & 9 \mathrm{~h} 30 & 20 \mathrm{~h} \\ \text { Samedi } 5 & 15 \mathrm{~h} 30 & 20 \mathrm{~h} \\ \text { Dimanche } 6 & 18 \mathrm{~h} & 24 \mathrm{~h} \\ \text { Lundi } 7 & 11 \mathrm{~h} & 24 \mathrm{~h} / 1 \mathrm{~h} \\ \text { Mardi } 8 & 15 \mathrm{~h} & 24 \mathrm{~h} \\ \text { Mercredi } 9 & 11 \mathrm{~h} & 22 \mathrm{~h} \\ \text { Jeudi } 10 & 12 \mathrm{~h} & 19 \mathrm{~h} \\ \text { Vendredi } 11 & 11 \mathrm{~h} & 15 \mathrm{~h} \text { puis } \\ & 19 \mathrm{~h} & 24 \mathrm{~h}\end{array}$

(24) Source: Claude Chetcuti, notes de terrain. 
Pour en sortir, la commission régionale de conciliation s'est réunie le 17 juin, mais on n'est arrivé à rien, bien évidemment. Je me souviens très bien qu'alors, j'avais fait une proposition en reprenant pratiquement mot à mot les termes de la médiation de Dupeyroux faite à Aulnay, mais que cela avait été repoussé par les grévistes. Ils estimaient que l'administration était partiale. Donc, le ministre a ressaisi Jean-Jacques Dupeyroux, et l'a renommé médiateur pour Poissy. Naturellement, il a repris quasiment ce qu'il avait écrit pour Citroën. Et comme c'était un médiateur et non pas un fonctionnaire, cela a été accepté. Comme chez Citroën (où les deux commissions pour les classifications et les salaires étaient présidées par Pierre Bois et moi-même), deux commissions ont été mises en place, l'une pour les libertés, présidée par Roger Salmon, qui était inspecteur général; l'autre, pour suivre les classifications, les salaires, etc. , sous la présidence du directeur départemental des Yvelines, qui venait d'être nommé, André Nutte. Après cela, le travail a repris. Mais, tant chez Citroën que chez Talbot, les commissions se sont réunies régulièrement de juillet ou septembre 1982 à mai 1983 pour traiter des problèmes que l'on ne pouvait pas résoudre immédiatement avec des résultats satisfaisants. $\mathrm{Au}$ début de l'année 1983, le ministre a tenu à recevoir lui-même le président de commission pour faire le point sur l'avancement des travaux.

Dans ces deux affaires, Citroën comme Talbot, un problème tenait à ce que l'interlocuteur de l'entreprise n'était pas dans l'entreprise. C'était manifeste chez Citroën, et à un degré moindre, chez Talbot: la représentation syndicale était très majoritairement CSL chez Citroën. Il y avait des embryons d'autres syndicats, dans le collège des agents de maitrise, mais pas chez les ouvriers. Chez Talbot, la CSL partageait son pouvoir avec un syndicat autonome nettement minoritaire, tandis que les syndicats confédérés étaient quantité négligeable. Finalement, la négociation pouvait difficilement s'engager dans l'entreprise, puisqu'il n'y avait pas d'interlocuteur dans la maison, de par la volonté des directions d'entreprise. Donc, à Aulnay comme à Poissy, une bonne partie de ceux qui venaient s'asseoir aux tables de négociation étaient soit des responsables fédéraux comme André Sainjon, responsable de la Fédération des métaux, soit des responsables de l'Union régionale ou de l'Union départementale de la métallurgie, comme à la CFDT ou à FO. Il fallait donc fatalement sortir de l'usine, et cela faisait un élément supplémentaire poussant à l'intervention de l'administration ».

\section{Administration et justice face aux conflits: parcours du XXe siècle}

Pour saisir les particularités de l'intervention de l'administration dans ces conflits, un retour en arrière n'est pas inutile. Dès 1892, sitôt mis en place Office du travail, Conseil supérieur du travail, inspection du travail généralisée, le législateur crée par la loi du 27 décembre 1892 un système de conciliation et d'arbitrage des conflits collectifs du travail, confié aux juges de paix. Cette solution n'est guère satisfaisante: le caractère facultatif de la procédure, l'absence de sanctions, le peu d'intérêt ou l'inexistence des partenaires sociaux appelés à représenter les parties ou, éventuellement, à être choisis comme arbitres, expliquent le peu d'efficacité du dispositif, bien que celui-ci demeure inscrit dans le Code du travail (articles 104 à 118 de l'ancien Livre IV) jusqu'à son abrogation par la loi du 11 février 1950. Jusqu'en 1936 la solution des conflits collectifs va être le fait d'amiables conciliateurs, élus locaux, notabilités acceptés par les parties. Avant la Première Guerre mondiale, les autorités locales estiment que ce rôle ne peut être tenu par les inspecteurs du travail, chargés d'une fonction de police, administrative ou judiciaire. Le rôle joué durant la guerre par les inspecteurs, affectés en grand nombre au ministère de l'Armement, qui les fait pénétrer au sein même de l'activité économique des entreprises participant à l'effort de guerre et régler les différents relatifs aux classifications et aux salaires, modifie la donne. Entre 1919 et 1936 les inspecteurs interviennent plus fréquemment, soit après le déclenchement du conflit, soit même en amont à la demande de l'une ou l'autre des parties.

1936 voit un tournant décisif. Dès la fin du mois de juin, le ministre Jean-Baptiste Lebas invite préfets et inspecteurs divisionnaires du travail à mobiliser les inspecteurs du travail pour faire reprendre, si nécessaire, le travail dans les usines encore nombreuses à être en grève. Un système de conciliation est mis en place dès l'été avant même que paraissent les textes les instituant définitivement (loi du 31 décembre 1936, décret du 16 janvier 1937). Mais le système se révèle lourd de par la multiplicité des instances, et peu efficace du fait que l'intervention immédiate de l'inspecteur du travail, d'un intervenant amiable ou encore d'une commission de conciliation ne s'impose pas aux parties. Dans la période de préparation à la guerre, une procédure plus contraignante est jugée nécessaire, celle de l'arbitrage obligatoire (loi du 4 mars 1938) intervenant lorsque les commissions de conciliation ont échoué, et qui fonctionne effectivement. La période de guerre (décret du 27 octobre 1939 relatif aux conventions collectives) et celle de l'immédiat après-guerre avec son régime de fixation des salaires (arrêtés Parodi) s'inscrit dans la même perspective et rétablit la négociation en même temps qu'elle libère les salaires.

La loi du 11 février 1950 relative aux conventions collectives et au règlement des conflits collectifs ne conserve l'arbitrage que comme une procédure facultative à l'initiative des parties. Il n'est pratiquement plus mis en œuvre, tandis que le passage devant les commissions de conciliation prend un caractère formel et peu efficace. Seul demeure le rôle informel, amiable 
de l'administration: préfet et inspecteur du travail doivent être avisés de toute grève, à charge pour eux (essentiellement pour le second, dès lors que l'ordre public n'est pas en jeu) d'intervenir afin de faciliter le règlement du conflit. C'est dans la période 19501975 que ce système fonctionne au mieux, la quasitotalité des conflits portant sur les salaires et leurs accessoires ou sur les classifications. Commissions de conciliation et surtout interventions informelles permettent aux intervenants amiables, généralement les inspecteurs du travail, de maintenir ou d'ouvrir le dialogue, ou encore de suggérer des solutions permettant à chacune des parties de ne pas perdre la face. L'intervention de l'administration porte aussi, et de façon importante, sur la gestion des fins de conflit: elle veille à l'application du protocole d'accord lorsqu'il y en a un et pèse de son autorité morale, comme le disent de nombreuses réponses ministérielles, pour limiter les sanctions envisagées par l'employeur, notamment à l'encontre des salariés ordinaires. Pour les salariés protégés, l'inspecteur veille au respect des dispositions les concernant, renforcées par la loi (ordonnance du 7 janvier 1959 et décret du même jour) ou la jurisprudence: l'arrêt Perrier de 1974 établit que la décision de l'inspecteur s'impose et que l'employeur ne peut plus demander la résolution d'un contrat de travail par les tribunaux (25).

Aux yeux du législateur, il s'avère toutefois nécessaire de prévoir pour les cas de conflits extrêmes une procédure intermédiaire entre l'arbitrage rejeté unanimement par les partenaires sociaux et la conciliation, celle de la médiation, instaurée en 1955. Désigné par l'autorité administrative, le médiateur dispose d'une grande liberté d'action et sa recommandation peut faire l'objet d'une publication en cas de refus par l'une ou l'autre des parties. Pourtant, après une première mise en œuvre peu après la promulgation de la loi, la procédure n'est plus utilisée, le ministère préférant nommer, dans les conflits importants qui ne trouvent pas de solution au niveau local, une personnalité généralement choisie parmi les hauts fonctionnaires ou les grands corps de l'État, mais sans recourir aux dispositions du Code du travail (articles L. 524-1 et suivants), jugées trop contraignantes pour les parties.

C'est dans ce cadre et cette pratique que doit être étudiée l'intervention de l'administration dans les conflits survenus au sein des usines Citroën et Talbot de la région parisienne en mai-juin 1982. Elle suit plusieurs interventions singulières, évoquées plus haut, telles que l'intervention en amont du juge des référés et de l'administration du travail pour faciliter la tenue d'élections libres (présence de membres du Conseil d'État en 1981; accord pour la présence d'inspecteurs du travail en 1982 chez Talbot, qui ne trouve pas effet compte tenu des circonstances).
À l'ouverture des conflits, elle se traduit par l'intervention rapide voire immédiate d'un fonctionnaire de haut rang (directeur régional du travail) chez Citroën ou du directeur des relations du travail luimême chez Talbot, puis par la présence du directeur régional du travail sur les sites considérés ou dans un lieu neutre pour faire se rencontrer les parties et organiser de nombreuses réunions. Tout ceci ne donne guère plus de résultat que le passage obligatoire mais vain devant la commission régionale de conciliation. Aux revendications classiques concernant salaires et classification s'ajoutent des revendications nouvelles, portant sur la liberté syndicale mais aussi la non-discrimination ethnique, la dignité des travailleurs et, pour la première fois, l'expression religieuse sur les lieux de travail. La complexité du dossier, qui interdit que puissent être trouvées des solutions immédiatement applicables, l'enchevêtrement des revendications amènent les membres du cabinet du ministre Jean Auroux (essentiellement Michel Praderie, directeur du cabinet et Gilbert Cornu, inspecteur général du travail chargé de mission auprès du ministre) à proposer d'utiliser la procédure de médiation, tombée dans l'oubli, et à avancer le nom de Jean-Jacques Dupeyroux, à l'indépendance et à la compétence reconnues par tous. Avec les résultats indiqués plus haut.

$*$
$* \quad *$

Ce survol appelle deux remarques conclusives. Tout d'abord, la loi prévoit qu'à l'occasion des conflits collectifs, l'inspecteur du travail, le préfet, voire le ministre, s'efforcent de mener une conciliation, de réunir les parties et d'œuvrer à l'émergence d'une solution. Par ailleurs, une pratique s'est instaurée de recourir aux juges des référés, d'abord à l'initiative des employeurs puis, plus récemment de la part des salariés, afin de peser sur la négociation. Cela constitue une singularité française, distincte de nombreux pays où les tribunaux du travail, quand ils existent, sont compétents tant pour les conflits collectifs qu'individuels. Ensuite, le basculement des conflits vers les problèmes de l'emploi renforce le rôle de l'administration, lorsqu'existe le régime du contrôle administratif des licenciements, mais aussi après sa suppression, avec la panoplie de mesures telles que préretraites, formation, etc. qu'il incombe à l'administration de mettre en œuvre, et qui maintiennent le poids de son intervention. 


\section{Bibliographie}

BeCKer J.-J. (1998), Crises et alternances, 1974-1995, collection «Nouvelle histoire de la France contemporaine», Paris, Le Seuil, pp. 270-273.

Benoit F. (1982), Citroën. Le printemps de la dignité, Paris, Messidor - Éditions Sociales, 1982.

Ewald, L'école des esclaves, Paris, éditions de la table ronde, 1983.

FAVRe D. (1998), Ni rouges ni jaunes. De la CGSI à la CSL, l'expérience du syndicalisme indépendant, éditions Midi moins le quart.

HatzFeld N. (2003), «"Faire tourner l'usine sans patron"? Entre rêve et lutte, quelques jours singuliers à Talbot-Poissy, en 1983 », in Georgi Franck, Autogestion. La dernière utopie? Paris, Publications de La Sorbonne, pp. 425-436.
Loubet J.-L. (2001), Histoire de l'automobile française, Paris, Le Seuil, pp. 387-396.

Loubet J.-L., Hatzfeld N., (2002), Les 7 vies de Poissy, Boulogne, éditions ETAI, p. 123.

Marty N. (2005), «Les conditions historiques d'un revirement de jurisprudence majeur dans l'histoire du droit de travail français: l'arrêt Perrier de la Cour de cassation (1974), in Dominique Barjot (sous la direction de), Le travail à l'époque contemporaine, Paris, éditions du CTHS, 2005, pp. 155-170.

PitTi L. (2002), Ouvriers algériens à Renault-Billancourt, de la guerre d'Algérie aux grèves d'OS des années 1970. Contribution à l'histoire sociale et politique des ouvriers étrangers en France, thèse de doctorat d'histoire, université Paris-VIII.

Trehel J. (1982), Un homme de Poissy, Paris, Éditions Sociales. 